\title{
Livšic theorem for diffeomorphism cocycles
}

\author{
Avila, Artur ; Kocsard, Alejandro ; Liu, Xiao-Chuan
}

\begin{abstract}
We prove the so called Livšic theorem for cocycles taking values in the group of $C^{1+}$ diffeomorphisms of any closed manifold of arbitrary dimension. Since no localization hypothesis is assumed, this result is completely global in the space of cocycles and thus extends the previous result of the Kocsard and Potrie (Comment Math Helv 91:39-64, 2016) to higher dimensions.
\end{abstract}

DOI: https://doi.org/10.1007/s00039-018-0454-y

Posted at the Zurich Open Repository and Archive, University of Zurich

ZORA URL: https://doi.org/10.5167/uzh-157561

Journal Article

Accepted Version

Originally published at:

Avila, Artur; Kocsard, Alejandro; Liu, Xiao-Chuan (2018). Livšic theorem for diffeomorphism cocycles. Geometric and Functional Analysis, 28(4):943-964.

DOI: https://doi.org/10.1007/s00039-018-0454-y 


\title{
LIVŠIC THEOREM FOR DIFFEOMORPHISM COCYCLES
}

\author{
ARTUR AVILA, ALEJANDRO KOCSARD, AND XIAO-CHUAN LIU
}

\begin{abstract}
We prove the so called Livšic theorem for cocycles taking values in the group of $C^{1+\beta}$-diffeomorphisms of any closed manifold of arbitrary dimension. Since no localization hypothesis is assumed, this result is completely global in the space of cocycles and thus extends the previous result of the second author and Potrie [KP16] to higher dimensions.
\end{abstract}

\section{INTRODUCTION}

Given a dynamical system $f: M \rightarrow M$ and a map $A: M \rightarrow G$, where $G$ denotes a topological group, a fundamental problem in the modern theory of dynamical systems consists in determining whether there exists a function $u: M \rightarrow G$ such that

$$
A(x)=u(f(x))(u(x))^{-1}, \quad \forall x \in M .
$$

Such an equation is usually called a cohomological equation and when it does admit a solution, the cocycle $A$ is said to be a coboundary for $f$.

A celebrated theorem of Livšic [Liv71, Liv72a, Liv72b] claims that when $f$ is a hyperbolic homeomorphism, the group $G$ is the additive real line $\mathbb{R}$ and $A$ is Hölder continuous, then there exists a continuous solution $u: M \rightarrow \mathbb{R}$ satisfying

$$
A(x)=u \circ f-f,
$$

if and only if

$$
\sum_{i=0}^{n-1} A\left(f^{i}(p)\right)=0, \quad \forall p \in \operatorname{Fix}\left(f^{n}\right), \quad \forall n \geqslant 1 .
$$

Many generalizations have been studied. Some of them consider more general dynamical systems on the base. For example, in [KK96] and [Wil13] cohomological equations over partially hyperbolic diffeomorphisms are studied. Other generalizations deal with cocycles taking values in more general groups (see for instance [dlLW10, dlLW10]).

In this paper, we consider the second kind of generalization. When $G$ is an arbitrary group, the above condition in Livšic theorem becomes

$$
A\left(f^{n-1}(p)\right) \cdots A(p)=e_{G},
$$

for any periodic point $p$ of period $n$, and where $e_{G}$ denotes the identity element of the group $G$.

Livšic himself has already considered more general groups in [Liv71]. In fact, in that work he deals with cocycles taking values in a topological groups admitting a complete bi-invariant distance (e.g. Abelian or compact groups), and in this case, the very same techniques that were applied to the real case (i.e. when $G=\mathbb{R}$ ) still work.

Looking for generalizations of these results to cocycles taking values in groups not admitting bi-invariant distances, many authors (for instance [Liv72a, dlLW10, dlLW11]) have applied "distortion control techniques" which consist in endowing $G$

Date: May 8, 2018. 
with a left-invariant distance and to estimate the distortion by right multiplication. However, in most of the cases this method alone only works under certain "localization hypothesis", i.e. assuming that the cocycle is sufficiently close to the constant identity cocycle in certain topology, and therefore, does not produce global results.

The first global Livšic like theorem for topological groups not admitting a biinvariant distance is due to Kalinin [Kal11], who proved Livšic theorem for linear cocycles substituting the localization hypothesis by some estimates of Lyapunov exponents.

In the infinite-dimensional case, that is when $G$ is a topological group of infinite dimension, Niţică and Török [NT95, NT96] got the first local results (i.e. under some localization hypothesis) for groups of diffeomorphisms of closed manifolds. Some extensions of these results were obtained by de la Llave and Windsor in [dlLW10], but always under certain localization hypotheses. Navas and Ponce dealt in [NP13] with the case of cocycles taking values in the group of analytic germs at the origin.

The first global Livšic type theorem for groups of diffeomorphisms was recently obtained by Potrie and the second author of this paper in [KP16]. There they got a sharp characterization of coboundaries in terms of Lyapunov exponents, introducing some new geometric arguments and avoiding the usage of control distortion techniques. With this characterization of coboundaries, that holds for groups of diffeomorphisms of manifolds of any dimension, they proved a Livšic type theorem for cocycles with values in the group of circle $C^{1}$-diffeomorphisms.

The main result of this paper is the so called Livšic theorem for Hölder cocycles taking values in the group of $C^{r}$-diffeomorphisms of a closed manifold, with $r>1$, extending the previous result of [KP16] to higher dimensions.

1.1. Main results. All along this work, $M$ will denote a compact metric space, $f: M \oslash$ a hyperbolic homeomorphism, $N$ a closed smooth manifold of dimension $q$ and $\operatorname{Diff}^{r}(N)$ the group of $C^{r}$-diffeomorphisms of $N$ (see $\S 2$ for details and definitions).

The main result of this work is the following

Theorem 1.1. Let $A: M \rightarrow \operatorname{Diff}^{1+\beta}(N)$ be a Hölder continuous cocycle, with $\beta>0$. Then, A satisfies the periodic orbit condition, i.e. ,

$$
A\left(f^{n-1}(p)\right) \cdots A(f(p)) A(p)=i d_{N}, \quad \forall p \in \operatorname{Fix}\left(f^{n}\right), \quad \forall n \geqslant 1,
$$

if and only if there exists a Hölder continuous map $u: M \rightarrow \operatorname{Diff}^{1+\beta}(N)$ such that

$$
A(x)=u(f(x)) \circ u(x)^{-1}, \quad \forall x \in M .
$$

As a consequence of Theorem 1.1 and some results due to de la Llave and Windsor [dlLW11], we get the Livšic theorem for groups of diffeomorphisms in higher regularity:

Corollary 1.2. Let $A: M \rightarrow \operatorname{Diff}^{r}(N)$ be a Hölder continuous cocycle, with $r>1$. Then, A satisfies the periodic orbit condition (3) if and only if there exists a Hölder continuous map $u: M \rightarrow \operatorname{Diff}^{r}(N)$ such that

$$
A(x)=u(f(x)) \circ u(x)^{-1}, \quad \forall x \in M .
$$

Acknowledgments. While this work was in preparation, we learned that M. Guysinsky was working on the very same problem, but applying rather different techniques. We would like to thank him for kindly sharing a draft of his work [Guy] with us while A.K. and X.L. were visiting Penn State University. A.K. is very indebted to Federico Rodríguez-Hertz for several insightful discussions and his hospitality during that visit. 
A.K. was partially supported by CNPq-Brazil (Bolsa de Produtividade em Pesquisa) and FAPERJ-Brazil (Jovem Cientista do Nosso Estado). X.L. was supported by CNPq-Brazil (Bolsa de Pós-Doutorado Júnior).

\section{Preliminaries and notations}

2.1. $C^{1+\beta}$-distance on groups of diffeomorphisms. All along this article $N$ will denote a compact Riemannian manifold and we shall write $d_{N}$ for its distance function induced by the Riemannian structure.

We shall consider the group of $C^{1+\beta}$-diffeomorphisms of $N$, which is denoted by $\operatorname{Diff}^{1+\beta}(N)$, endowed with the distance function $d_{C^{1+\beta}}$ which is defined as follows: first we define the $d_{C^{0}}$ distance by

$$
d_{C^{0}}(f, g):=\max \{d(f(x), g(x)): x \in N\}, \quad \forall f, g \in \operatorname{Diff}^{1+\beta}(N) .
$$

Then, since $N$ is compact, there is finite atlas $\left\{\phi_{i}: U_{i} \subset N \rightarrow \mathbb{R}^{q}, i=1, \ldots, m\right\}$ and compact sets $K_{i} \subset U_{i}$ such that $\bigcup_{i=1}^{n} K_{i}=N$. So, given any pair of diffeomorphisms $f, g \in \operatorname{Diff}^{1+\beta}(N)$ satisfying

$$
f \circ g^{-1}\left(K_{i}\right), g \circ f^{-1}\left(K_{i}\right) \subset U_{i}, \quad \forall i \in\{1, \ldots, m\},
$$

we define the distance $d_{C^{1}}$ by

$$
\begin{aligned}
d_{C^{1}}(f, g):=d_{C^{0}}(f, g) & +\max _{1 \leqslant i \leqslant m}\left\{\max _{x \in \phi_{i}\left(K_{i}\right)}\left\|D\left(\phi_{i} \circ f \circ g^{-1} \circ \phi_{i}^{-1}(x)\right)\right\|\right\} \\
& +\max _{1 \leqslant i \leqslant m}\left\{\max _{x \in \phi_{i}\left(K_{i}\right)}\left\|D\left(\phi_{i} \circ g \circ f^{-1} \circ \phi_{i}^{-1}(x)\right)\right\|\right\} .
\end{aligned}
$$

Then, we define the distance $d_{C^{1+\beta}}$ by

$$
\begin{aligned}
& d_{C^{1+\beta}}(f, g):=d_{C^{1}}(f, g)+ \\
& \max _{1 \leqslant i \leqslant m}\left\{\max _{x, y \in \phi_{i}\left(K_{i}\right), x \neq y} \frac{\left\|D\left(\phi_{i} \circ f \circ g^{-1} \circ \phi_{i}^{-1}(x)\right)-D\left(\phi_{i} \circ f \circ g^{-1} \circ \phi_{i}^{-1}(y)\right)\right\|}{\|x-y\|^{\beta}}\right\}+ \\
& \max _{1 \leqslant i \leqslant m}\left\{\max _{x, y \in \phi_{i}\left(K_{i}\right), x \neq y} \frac{\left\|D\left(\phi_{i} \circ g \circ f^{-1} \circ \phi_{i}^{-1}(x)\right)-D\left(\phi_{i} \circ g \circ f^{-1} \circ \phi_{i}^{-1}(y)\right)\right\|}{\|x-y\|^{\beta}}\right\} .
\end{aligned}
$$

Finally, we define $d_{C^{1}}(f, g)=d_{C^{0}}(f, g)+1$ and $d_{C^{1+\beta}}(f, g)=d_{1} C^{1}(f, g)+1$, whenever condition (4) does not hold.

2.2. Hyperbolic homeomorphisms. Given a compact metric space $(M, d)$, a homeomorphism $f: M ๖$ and an arbitrary point $x \in M$, let us consider the local stable and unstable sets at $x$ given by

$$
\begin{aligned}
& W_{\varepsilon}^{s}(x):=\left\{y \in M: d\left(f^{n}(x), f^{n}(y)\right) \leqslant \varepsilon, \forall n \geqslant 0\right\} \\
& W_{\varepsilon}^{u}(x):=\left\{y \in M: d\left(f^{-n}(x), f^{-n}(y)\right) \leqslant \varepsilon, \forall n \geqslant 0\right\},
\end{aligned}
$$

where $\varepsilon$ is any positive real number.

Definition 2.1. The homeomorphism $f$ is said to be hyperbolic whenever it is bi-Lipschitz (i.e. $f$ and $f^{-1}$ are Lipschitz), transitive and there exist constants $\varepsilon, \delta, K_{0}, \tau>0$ and continuous real functions $\nu_{s}, \nu_{u}: M \rightarrow(0, \infty)$ such that the following properties hold:

(i) $d\left(f\left(y^{\prime}\right), f\left(y^{\prime \prime}\right)\right) \leqslant \nu_{s}(y) d\left(y^{\prime}, y^{\prime \prime}\right)$ for any $y^{\prime}, y^{\prime \prime} \in W_{\varepsilon}^{s}(y)$ and any $y \in M$;

(ii) $d\left(f^{-1}\left(y^{\prime}\right), f^{-1}\left(y^{\prime \prime}\right)\right) \leqslant \nu_{u}(y) d\left(y^{\prime}, y^{\prime \prime}\right)$ for any $y^{\prime}, y^{\prime \prime} \in W_{\varepsilon}^{u}(y)$ and any $y \in M$;

(iii) $\nu_{s}^{(n)}(y)=\nu_{s}\left(f^{n-1}(y)\right) \cdots \nu_{s}(y) \leqslant K_{0} e^{-\tau n}$ for any $y \in M$ and any $n \geqslant 1$;

(iv) $\nu_{u}^{(n)}(y)=\nu_{u}\left(f^{-(n-1)}(y)\right) \cdots \nu_{u}(y) \leqslant K_{0} e^{-\tau n}$ for any $y \in M$ and any $n \geqslant 1$.

(v) if $d\left(y, y^{\prime}\right)<\delta$, then $W_{\varepsilon}^{u}(y) \cap W_{\varepsilon}^{s}\left(y^{\prime}\right)$ consists of exactly one point, denoted by $\left[y, y^{\prime}\right]$, which depends continuously on $\left(y, y^{\prime}\right)$, 
A fundamental property about hyperbolic homeomorphisms is given by the so called Anosov closing lemma (see for instance [KH96, Theorem 6.4.15]):

Lemma 2.2. If $f: M \bigcirc$ is a hyperbolic homeomorphism, then there exist constants $C, \delta>0$ such that for any $x \in M$ and any $n \geqslant 1$ such that $d\left(x, f^{n}(x)\right)<\delta$, there exists a unique periodic point $p \in \operatorname{Fix}\left(f^{n}\right)$ satisfying

$$
d\left(f^{i}(x), f^{i}(p)\right) \leqslant C d\left(x, f^{n}(x)\right) e^{-\tau \min (i, n-i)} \text { for any } i=0, \cdots, n,
$$

where $\tau$ is the constant given by Definition 2.1.

Moreover, if we define $y:=[p, x]$, it holds

$$
d\left(f^{i}(x), f^{i}(y)\right) \leqslant C d\left(x, f^{n}(x)\right) e^{-\tau i},
$$

and

$$
d\left(f^{i}(p), f^{i}(y)\right)<C d\left(x, f^{n}(x)\right) e^{-\tau(n-i)},
$$

for any $i=0,1, \ldots, n$.

2.3. Cocycles and coboundaries. Let $G$ be a topological group and $f: M ๖$ be a hyperbolic homeomorphism. Every continuous map $A: M \rightarrow G$ shall be considered as a $G$-cocycle over $f$, i.e. we will use the following notation: given any $n \in \mathbb{Z}$, we write

$$
A^{n}(x):= \begin{cases}e_{G}, & \text { if } n=0 \\ A\left(f^{n-1}(x)\right) \cdots A(x), & \text { if } n \geqslant 1, \\ A\left(f^{n}(x)\right)^{-1} \cdots A\left(f^{-1}(x)\right)^{-1}, & \text { if } n<0 .\end{cases}
$$

It is important to notice that any $G$-cocycle over $f: M \diamond$ naturally induces a skew-product map $F=(f, A): M \times G ๖$ given by

$$
(f, A)(x, g):=(f(x), A(x) g), \quad \forall(x, g) \in X \times G .
$$

Observe that in this case, it holds $(f, A)^{n}(x, g)=\left(f^{n}(x), A^{n}(x) g\right)$, for every $n \in \mathbb{Z}$, and any $(x, g) \in M \times G$.

Definition 2.3. A $G$-cocycle $A$ is called a coboundary whenever there exists a continuous mapping $u: M \rightarrow G$ satisfying

$$
A(x)=u(f(x)) \cdot u(x)^{-1}, \quad \forall x \in M .
$$

An obvious, yet extremely important, family of obstructions for a $G$-cocycle to be a coboundary is given by periodic orbits of $f$. In fact, if $A$ is a coboundary, it necessarily holds

$$
A^{n}(p)=e_{G}, \quad \forall n \in \mathbb{N}, \forall p \in \operatorname{Fix}\left(f^{n}\right) .
$$

In this paper we will say that a $G$-cocycle $A$ satisfies the periodic orbit condition ( $P O C$ for short) whenever property (7) holds.

2.4. Fibered Lyapunov exponents. In this work we are mainly interested in the study of $G$-cocycles with $G<\operatorname{Diff}^{r}(N)$, where $N$ is a closed smooth manifold.

From now on and till the end of the paper we will suppose that $N$ endowed with a Riemannian structure $\langle\cdot, \cdot\rangle$, and the norm induced by the Riemannian structure will be denoted by $\|\cdot\|$.

In this case, when $G$ is a subgroup of the $\operatorname{Diff}^{r}(N)$, we can slightly modify the skew-product given by (5) defining $F=(f, A): M \times N \bigcirc$ by

$$
F(x, y):=(f(x), A(x) y), \quad \forall(x, y) \in M \times N .
$$

Observe that the map $F$ is $C^{r}$ along the vertical fibers. So, we can consider the "partial derivative of $F$ respect to the $N$ coordinate", defining the linear cocycle over $F$ given by

$$
\partial F_{(x, y)}^{n} v:=D\left(A^{n}(x)\right)(y)(v), \quad \forall x \in M, \forall y \in N, \forall v \in T_{y} N,
$$


and any $n \in \mathbb{Z}$.

In this way we can apply Oseledets theorem to guarantee that, for every ergodic $F$-invariant probability measure $\mu$, there exist $k \leqslant \operatorname{dim} N$, real numbers $\lambda_{1}^{\prime}>\lambda_{2}^{\prime}>$ $\ldots>\lambda_{k}^{\prime}$ and a measurable $F$-invariant set $\Lambda \subset M \times N$ with $\mu(\Lambda)=1$, such that for every $z=(x, y) \in \Lambda$ there exists a linear splitting $T_{y} N=E_{z}^{1} \oplus E_{z}^{2} \oplus \ldots E_{z}^{k}$ which measurably depends on $z$, satisfies $\partial F_{z}\left(E_{z}^{i}\right)=E_{F(z)}^{i}$, for every $z \in \Lambda$ and every $1 \leqslant i \leqslant k$, and

$$
\lambda_{i}^{\prime}:=\lim _{n \rightarrow \pm \infty} \frac{1}{n} \log \left\|\partial F_{z}^{n}(v)\right\|, \quad \forall z \in \Lambda, \quad \forall v \in E_{z}^{i} \backslash\{0\}, \forall i \leqslant k .
$$

2.5. Lyapunov exponents and coboundaries. As it was already shown in [KP16], when dealing with cocycle over hyperbolic homeomorphisms with values in groups of diffeomorphisms there is a very tight relation between Lyapunov exponents and coboundaries. In fact, the main result of [KP16] is the following:

Theorem 2.4 (Theorem 3.1 in [KP16]). Let $f: M$ be a hyperbolic homeomorphism and $A: M \rightarrow \operatorname{Diff}^{1}(N)$ be a Hölder continuous cocycle over $f$ satisfying the POC given by (7).

Then the following statements are equivalent:

(1) Every Lyapunov exponent given by (10) with respect to any F-invariant measure vanish, i.e. it holds

$$
\lim _{n \rightarrow \pm \infty} \frac{1}{n} \log \left\|\partial F_{z}^{n}\right\|=0, \quad \forall z \in M \times N ;
$$

(2) The cocycle $A$ is a coboundary, i.e. there exists a Hölder continuous map $u: M \rightarrow \operatorname{Diff}^{1}(N)$ such that

$$
A(x)=u(f(x)) \circ u(x)^{-1}, \quad \forall x \in M .
$$

Remark. We should note that, for our main statement, we have to consider $C^{1+\beta}$ topology instead of $C^{1}$ topology as in the above statement. The reason is that the proof involves techniques from Pesin theory.

So, in order to show Theorem 1.1 it is enough to prove the following

Theorem 2.5. If $f: M \bigcirc$ is a hyperbolic homeomorphism and $A: M \rightarrow \operatorname{Diff}^{1+\beta}(N)$ is a Hölder cocycle satisfying POC, then

$$
\lim _{n \rightarrow \pm \infty} \frac{1}{n} \log \left\|\partial F_{z}^{n}\right\|=0, \quad \forall z \in M \times N .
$$

It is worth mentioning that in [KP16] it is considered a slightly more general setting, dealing with $\grave{a}$ priori arbitrary fiber bundles. There it is shown that the fiber bundle is à posteriori trivial whenever the corresponding fiber bundle map turns to be a coboundary (see $\S 2.6$ in [KP16] for more details).

Since all our techniques are either local or semi-local, we could have worked in the fiber bundle category, getting the very same results. However, in order to avoid cumbersome unnecessary technicalities we have decided to present all our work in product spaces, i.e. à priori trivial fiber bundles.

\section{Some LineAr ALgEBRAiC LEMmas}

Let us consider $\mathbb{R}^{d}$ endowed with its usual Euclidean structure. Let us denote the closed ball of radius $r$ and centered at the origin by $B_{r}:=\left\{x \in \mathbb{R}^{d}:\|x\| \leqslant r\right\}$ and the sphere of radius $r$ by $\mathbb{S}_{r}:=\left\{x \in \mathbb{R}^{d}:\|x\|=r\right\}$.

Given an ellipsoid $E \subset \mathbb{R}^{d}$, we shall write $r_{1}(E) \geqslant r_{2}(E) \geqslant \cdots \geqslant r_{d}(E)$ for the ordered lengths of its semi-axes.

Our first result of this section is the following elementary 
Lemma 3.1. If $E, F \subset \mathbb{R}^{d}$ are two ellipsoids centered at the origin and such that $E$ is contained in the interior of $F$, then it holds

$$
r_{i}(E) \leqslant r_{i}(F), \quad \forall i \in\{1, \ldots, d\} .
$$

Proof. Let $\left\{e_{1}, e_{2}, \ldots, e_{d}\right\}$ and $\left\{f_{1}, f_{2}, \ldots, f_{d}\right\}$ be two orthonormal bases of $\mathbb{R}^{d}$ such that the directions of $e_{i}$ and $f_{i}$ coincide with the direction of the $i^{\text {th }}$-axis of $E$ and $F$, respectively.

First notice that, since $E$ is contained in the interior of $F$, it clearly holds $r_{1}(E) \leqslant r_{1}(F)$. Reasoning by contradiction, let us suppose there exists $j \in$ $\{2, \ldots, d\}$ such that $r_{i}(E) \leqslant r_{i}(F)$, for every $i \leqslant j-1$ and $r_{j}(E)>r_{j}(F)$. Let $V_{E, j}:=\operatorname{span}\left\{e_{1}, \ldots, e_{j}\right\}$ and $V_{F}^{j}:=\operatorname{span}\left\{f_{j}, \ldots, f_{d}\right\}$. Notice that $\|v\| \geqslant$ $r_{j}(E)$, for all $v \in E \cap V_{E, j}$; and $\|v\| \leqslant r_{j}(F)$, for every $v \in F \cap V_{F}^{j}$. However, $\operatorname{dim} V_{E, j}+\operatorname{dim} V_{F}^{j}=j+d-j+1=d+1$. So, there exists some $v \in E \cap V_{E, j} \cap V_{F}^{j}$ and consequently, $\|v\| \geqslant r_{j}(E)>r_{j}(F)$, contradicting the fact that $E$ is contained in the interior of $F$.

For any $A \in \mathrm{GL}(d, \mathbb{R})$, the image of the unit sphere $A\left(\mathbb{S}_{1}\right)$ is an ellipsoid centered at the origin, and the singular values of $A$ are defined by

$$
\sigma_{i}(A):=r_{i}\left(A\left(\mathbb{S}_{1}\right)\right), \quad \text { for } i \in\{1, \ldots, d\} .
$$

It is well-known that $\sigma_{i}(A)^{2}$ is an eigenvalue for $A A^{t}$, where $A^{t}$ denotes the transpose of $A$.

Our first perturbative result is the following:

Lemma 3.2. Let $\left(A_{n}\right)_{n \geqslant 1}$ be a sequence of matrices in $\operatorname{GL}(d, \mathbb{R})$ and suppose there exist real numbers $\lambda_{1} \geqslant \lambda_{2} \geqslant \cdots \geqslant \lambda_{d}$ and $\delta>0$ such that

$$
\left|\frac{1}{n} \log \left(\sigma_{i}\left(A_{n}\right)\right)-\lambda_{i}\right| \leqslant \frac{\delta}{2}, \quad \forall n \geqslant 1, \quad \forall i \in\{1, \ldots, d\} .
$$

Then, given any compact set $K \subset \mathrm{GL}(d, \mathbb{R})$, there exists $N=N(K)>0$ such that for every pair of sequences $\left(C_{n}\right)_{n \geqslant 1},\left(D_{n}\right)_{n \geqslant 1} \subset K$, it holds

$$
\left|\frac{1}{n} \log \left(\sigma_{i}\left(C_{n} A_{n} D_{n}\right)\right)-\lambda_{i}\right| \leqslant \delta, \quad \forall n \geqslant N,
$$

and every $i \in\{1, \ldots, d\}$.

Proof. Since $K$ is compact, there exists $\ell>1$ such that

$$
\max \left\{\|C\|,\left\|C^{-1}\right\|\right\} \leqslant \ell, \quad \forall C \in K .
$$

So, if $E$ is an arbitrary ellipsoid centered at the origin, then we have $\frac{1}{\ell} E \subset$ $C(E) \subset \ell E$, and by Lemma 3.1 it holds

and every $i$.

$$
\frac{1}{\ell} r_{i}(E) \leqslant r_{i}(C(E)) \leqslant \ell r_{i}(E), \quad \forall C \in K
$$

Consequently, for any $n \geqslant 1$ it clearly holds

$$
\frac{1}{\ell^{2}} \sigma_{i}\left(A_{n}\right) \leqslant \sigma_{i}\left(C_{n} A_{n} D_{n}\right) \leqslant \ell^{2} \sigma_{i}\left(A_{n}\right), \quad \forall n \geqslant 1,
$$

and every $i$.

So,

$$
\begin{aligned}
-\frac{2 \log \ell}{n}+\frac{1}{n} \log \sigma_{i}\left(A_{n}\right)-\lambda_{i} & \leqslant \frac{1}{n} \log \left(\sigma_{i}\left(C_{n} A_{n} D_{n}\right)\right)-\lambda_{i} \\
& \leqslant \frac{2 \log \ell}{n}+\sigma_{i}\left(A_{n}\right)-\lambda_{i},
\end{aligned}
$$

for every $n$ and any $i$, and taking $N:=\frac{4 \log \ell}{\delta}$ the lemma is proved. 
Lemma 3.3. Let $\mathbb{R}^{d}=\bigoplus_{i=1}^{k} E^{i}$ be an orthogonal linear decomposition and write $d_{i}:=\operatorname{dim} E^{i}$. Consider real numbers $\lambda_{1}^{\prime}>\lambda_{2}^{\prime}>\ldots>\lambda_{k}^{\prime}$, define

$$
\kappa:=\frac{1}{2} \min _{1 \leqslant i \leqslant k-1}\left\{\lambda_{i}^{\prime}-\lambda_{i+1}^{\prime}\right\}
$$

and let $\delta$ be any number satisfying $0<\delta<\kappa / 2$.

Let $\left(A_{n}\right)_{n \geqslant 1} \subset \mathrm{GL}(d, \mathbb{R})$ be a sequence of matrices such that

$$
A_{n}\left(E^{i}\right)=E^{i}
$$

and

$$
\left\|\left.A_{n}\right|_{E^{i}}\right\|,\left\|\left.A_{n}^{-1}\right|_{E^{i}}\right\|^{-1} \in\left(e^{\lambda_{i}^{\prime}-\frac{\delta}{4}}, e^{\lambda_{i}^{\prime}+\frac{\delta}{4}}\right), \quad \forall n \geqslant 1,
$$

and every $i \in\{1, \ldots, k\}$.

Then, there exist $\alpha_{1}=\alpha_{1}(\delta)>0$ so that for every $\left(C_{n}\right)_{n \geqslant 1} \subset \operatorname{GL}(d, \mathbb{R})$ satisfying

$$
\left\|A_{n}-C_{n}\right\|<\alpha_{1}, \quad \forall n \geqslant 1
$$

it holds

$$
\left|\frac{1}{n} \log \sigma_{i}\left(A^{(n)}\right)-\frac{1}{n} \log \sigma_{i}\left(C^{(n)}\right)\right|<\delta, \quad \forall n \geqslant 1, \forall i \in\{1, \ldots, k\},
$$

where $A^{(n)}:=\prod_{j=1}^{n} A_{j}$ and $C^{(n)}:=\prod_{j=1}^{n} C_{j}$.

Proof. Given any $v \in \mathbb{R}^{d}$, we write $v=\sum_{i=1}^{k} v^{i}$ for its unique decomposition with $v^{i} \in E^{i}$, for each $i$.

For each $j \in\{1, \ldots, k-1\}$ and $\gamma>0$, let us consider the cones

$$
K_{\gamma}^{j}:=\left\{v \in \mathbb{R}^{d}:\left\|\sum_{i \geqslant j+1} v^{i}\right\|<\gamma\left\|\sum_{i \leqslant j} v^{i}\right\|\right\},
$$

and

$$
K_{j, \gamma}:=\left\{v \in \mathbb{R}^{d}:\left\|\sum_{i \leqslant j} v^{i}\right\|<\gamma\left\|\sum_{i \geqslant j+1} v^{i}\right\|\right\} .
$$

For each $j \in\{1, \ldots, k-1\}$ and any $n$, since we are assuming the linear decomposition $\mathbb{R}^{d}=\bigoplus_{i} E^{i}$ is orthogonal, it holds

$$
\begin{aligned}
\left\|A_{n} \sum_{i \leqslant j} v^{i}\right\|^{2} & =\sum_{i \leqslant j}\left\|A_{n} v^{i}\right\|^{2} \geqslant\left(e^{\lambda_{j}^{\prime}-\delta / 2}\right)^{2}\left\|\sum_{i \leqslant j} v^{i}\right\|^{2} \\
& >\left(e^{\lambda_{j+1}^{\prime}+\kappa}\right)^{2}\left\|\frac{1}{\gamma} \sum_{i \geqslant j+1} v^{i}\right\|^{2}>\left(\frac{e^{\kappa-\delta / 2}}{\gamma}\right)^{2}\left\|A_{n} \sum_{i \geqslant j+1} v^{i}\right\|^{2},
\end{aligned}
$$

for every $v \in K_{\gamma}^{j}$. Analogously, for every $v \in K_{j, \gamma}$ we get

$$
\begin{aligned}
\left\|A_{n}^{-1} \sum_{i \leqslant j} v^{i}\right\|^{2} & =\sum_{i \leqslant j}\left\|A_{n}^{-1} v^{i}\right\|^{2} \leqslant\left(e^{\lambda_{j}^{\prime}-\delta / 3}\right)^{-2}\left\|\sum_{i \leqslant j} v^{i}\right\|^{2} \\
& <\left(e^{\lambda_{j+1}^{\prime}+\kappa+\delta / 12}\right)^{-2}\left\|\gamma \sum_{i \geqslant j+1} v^{i}\right\|^{2}<\left(\frac{\gamma}{e^{\kappa-\delta / 4}}\right)^{2}\left\|A_{n}^{-1} \sum_{i \geqslant j+1} v^{i}\right\|^{2} .
\end{aligned}
$$

This implies that

$$
A_{n}\left(K_{\gamma}^{j}\right) \subset K_{\gamma e^{-\kappa+\delta / 4}}^{j}, \quad \text { and } \quad A_{n}^{-1}\left(K_{j, \gamma}\right) \subset K_{j, \gamma e^{-\kappa+\delta / 4}},
$$

and for every $\gamma>0$, each $j \in\{1, \ldots, d-1\}$ and any $n \geqslant 1$.

Then let us fix $\gamma>0$ sufficiently small such that

$$
\left\|A_{n} v\right\| \leqslant e^{\lambda_{j+1}^{\prime}+\delta / 3}\|v\|, \quad \text { and } \quad\left\|A_{n}^{-1} u\right\| \geqslant e^{\lambda_{j}^{\prime}-\delta / 3}\|u\|
$$


for every $j<k$, any $v \in K_{j, \gamma}$, all $u \in K_{\gamma}^{j}$ and every $n \geqslant 1$.

After fixing $\gamma$ as above, and taking into account (14) and (15), we choose $\alpha_{1}>0$ small enough such that for any sequence of matrices $\left(C_{n}\right)_{n \geqslant 1} \subset \mathrm{GL}(d, \mathbb{R})$ satisfying $\left\|A_{n}-C_{n}\right\|<\alpha_{1}$ for every $n$, it holds

$$
C_{n}\left(K_{\gamma}^{j}\right) \subset K_{\gamma e^{-\kappa+\delta / 2}}^{j}, \quad \text { and } \quad C_{n}^{-1}\left(K_{j, \gamma}\right) \subset K_{j, \gamma e^{-\kappa+\delta / 2}} .
$$

and

$$
\left\|C_{n} v\right\| \leqslant e^{\lambda_{j+1}^{\prime}+\delta / 2}\|v\|, \quad \text { and } \quad\left\|C_{n}^{-1} u\right\| \geqslant e^{\lambda_{j}^{\prime}-\delta / 2}\|u\|,
$$

for every $j \in\{1, \ldots, d-1\}$, any $n \geqslant 1$, every $v \in K_{j, \gamma}$ and all $u \in K_{\gamma}^{j}$.

Then, for any such sequence $\left(C_{n}\right)_{n \geqslant 1}$, let us define

$$
H_{k}:=\bigcap_{n \geqslant 1}\left(C^{(n)}\right)^{-1}\left(\overline{K_{k-1, \gamma}}\right) .
$$

By classical arguments one can easily show that $H_{k}$ is a linear subspace satisfying $\operatorname{dim} H_{k}=\operatorname{dim} E^{k}$. Putting together (16), (18) and (19) one can easily verify that

$$
e^{n\left(\lambda_{k}-\delta / 2\right)}\|v\| \leqslant\left\|C^{(n)} v\right\| \leqslant e^{n\left(\lambda_{k}^{\prime}+\delta / 2\right)}\|v\|, \quad \forall v \in H^{k} .
$$

On the other hand, for every $v \in \mathbb{R}^{d} \backslash H^{k}$, there exists $N \in \mathbb{N}$ such that $C^{(n)}(v) \in$ $K_{\gamma}^{k-1}$, and thus one gets

$$
\liminf _{n \rightarrow+\infty} \frac{1}{n} \log \left\|C^{(n)}(v)\right\| \geqslant \lambda_{k-1}^{\prime}-\delta>\lambda_{k}^{\prime}+\delta .
$$

Then, we define

$$
H_{k-1}:=\bigcap_{n \geqslant 1}\left(C^{(n)}\right)^{-1}\left(\overline{K_{k-2, \gamma}}\right),
$$

and it can be shown that $H_{k-1} \supset H_{k}$ is a linear subspace satisfying $\operatorname{dim} H_{k-1}=$ $\operatorname{dim} E^{k}+\operatorname{dim} E^{k-1}$, and by a similar argument to that used above, we get

$$
e^{n\left(\lambda_{k-1}^{\prime}-\delta / 2\right)}\|v\| \leqslant\left\|C^{(n)}(v)\right\| \leqslant e^{n\left(\lambda_{k-1}^{\prime}+\delta / 2\right)}\|v\|, \quad \forall v \in H_{k-1} \backslash H_{k} .
$$

Inductively one defines the flag $\mathbb{R}^{d}=H_{1} \supset H_{2} \supset \cdots \supset H_{k}$ such that for any $v \in H_{j} \backslash H_{j+1}$, it holds

$$
\lambda_{j}^{\prime}-\frac{\delta}{2} \leqslant \liminf _{n \rightarrow+\infty} \frac{1}{n} \log \left\|C^{(n)}(v)\right\| \leqslant \limsup _{n \rightarrow+\infty} \frac{1}{n} \log \left\|C^{(n)}(v)\right\| \leqslant \lambda_{j}^{\prime}+\frac{\delta}{2} .
$$

Finally, by (11) and (12) we know that for each $i \in\{1, \ldots, d\}$, there exists $j \in\{1, \ldots, k\}$ such that

$$
\left|\frac{1}{n} \sigma_{i}\left(A^{(n)}\right)-\lambda_{j}^{\prime}\right| \leqslant \frac{\delta}{2},
$$

and by (20) we also know that

$$
\left|\frac{1}{n} \sigma_{i}\left(C^{(n)}\right)-\lambda_{j}^{\prime}\right| \leqslant \frac{\delta}{2} .
$$

Then, (13) is consequence of last two estimates and the lemma is proved. 


\section{Fiber-wise Pesin Theory and Fake Invariant Sets}

The purpose of this section consists introducing the so called fake invariant sets, which are something like a "finite-time stable and unstable sets" and are mainly inspired by fake invariant foliations of Burns and Wilkinson [BW10] and pseudohyperbolic families of Hirsch, Pugh and Shub [HPS77]. We start stating a fiber-wise version of some classical notions and results due to Pesin, and we shall just sketch their proof.

Let $f: M \circlearrowleft$ be a hyperbolic homeomorphism on the compact metric space $\left(M, d_{M}\right), A: M \rightarrow \operatorname{Diff}^{1+\beta}(N)$ a $C^{\beta}$-cocycle, for some $\beta>0$, and $F: M \times N b$ be the induced skew-product given by (8). Let $d_{N}$ be the distance function on $N$ induced by its Riemannian structure. We shall consider the space $M \times N$ endowed with the distance $d$ given by

$$
d\left(z, z^{\prime}\right):=\sqrt{d_{M}\left(x, x^{\prime}\right)^{2}+d_{N}\left(y, y^{\prime}\right)^{2}}, \quad \forall z=(x, y), z^{\prime}=\left(x^{\prime}, y^{\prime}\right) \in M \times N .
$$

Notice that, since $A$ is a $C^{\beta}$-Hölder map, if $d_{C^{1+\beta}}$ denotes a distance on $\operatorname{Diff}^{1+\beta}(N)$ inducing the uniform $C^{1+\beta}$-topology, then there exists a real constant $K>0$ such that

$$
d_{C^{1+\beta}}\left(A(x), A\left(x^{\prime}\right)\right) \leqslant K d_{M}\left(x, x^{\prime}\right)^{\beta}, \quad \forall x, x^{\prime} \in M,
$$

where we use the distance $d_{C^{1+\beta}}$ defined in Section 2.1.

4.1. Fiber-wise Pesin charts. Consider the linear cocycle $\partial F: M \times T N Ð$ given by (9), which is in fact a fiber bundle map over $F$. Let us fix an arbitrary ergodic $F$-invariant probability measure $\mu$.

Then, there exists a finite collection of open subsets $\Delta_{1}, \Delta_{2}, \ldots, \Delta_{k_{0}}$ of $N$ such that

$$
\mu\left(M \times\left(N \backslash \bigcup_{i=1}^{k_{0}} \Delta_{i}\right)\right)=0,
$$

and the tangent bundle $\left.T N\right|_{\Delta_{i}}$ is trivial, i.e. is diffeomorphic to $\Delta_{i} \times \mathbb{R}^{q}$, for every $i \in\left\{1, \ldots, k_{0}\right\}$, where $q$ denotes the dimension of $N$. Via a smooth trivialization of the tangent bundle $T N$ over each $\Delta_{i}$, we can identify, up to $\mu$-measure zero, the derivative bundle map $\partial F: M \times T N \bigcirc$ with a linear cocycle over $F: M \times N \sqsubseteq$ (for details see [KH96, Supplement, §2.b]).

In other words, from now on we shall simply assume that the bundle map $\partial F$ is represented by a linear cocycle

$$
B(z):=D A(x)(y) \in \mathrm{GL}(q, \mathbb{R}), \quad \forall z=(x, y) \in M \times N,
$$

which is well-defined $\mu$-almost everywhere.

Now, let $r>0$ be the injectivity radius of the Rimeannian manifold $N$. For any linear cocycle $L: M \times N \rightarrow \operatorname{GL}(q, \mathbb{R})$, let us define the injective map $\Phi_{z}: B(y, r) \rightarrow$ $\mathbb{R}^{q}$ by

$$
\Phi_{z}\left(y^{\prime}\right):=L(z) \circ \exp _{y}^{-1}\left(y^{\prime}\right), \quad \forall y^{\prime} \in B(y, r),
$$

where $B(y, r) \subset\{x\} \times N$ is the ball of radius $r$ and center $y$ with respect to the distance $d_{N}$.

For $r^{\prime}>0$ sufficiently small (and smaller than $r$ in the previous paragraph), from now on, $z=(x, y)$ and $z^{\prime}=\left(x^{\prime}, y\right)$ will denote points of $M \times N$, where $x^{\prime} \in B_{M}\left(x, r^{\prime}\right)$. Now, we are interested in the cocycle $L=B$, the derivative cocycle (22), $\Phi_{z}$ will be the corresponding function defined in (23), and hence, we can define the map $h_{z, z^{\prime}}: B\left(0, r^{\prime}\right) \subset \mathbb{R}^{q} \rightarrow \mathbb{R}^{q}$ by

$$
h_{z, z^{\prime}}(v):=\Phi_{F(z)} \circ \operatorname{pr}_{2} \circ F\left(x^{\prime}, \Phi_{z}^{-1}(v)\right), \quad \forall v \in B\left(0, r^{\prime}\right) .
$$


Let $\lambda_{1}^{\prime}>\cdots>\lambda_{k}^{\prime}$ be the Lyapunov exponents of the linear cocycle $B$ with respect to $\mu$, where each exponent $\lambda_{j}^{\prime}$ has multiplicity $n_{j}, j=1, \ldots k$. The following result is a slight generalization of the classical construction of Pesin charts:

Theorem 4.1. For every $\eta>0$, there exist a measurable set $\Lambda_{\eta} \subset M \times N$ with full $\mu$-measure and a tempered map $C_{\eta}: M \times N \rightarrow \operatorname{GL}(q, \mathbb{R})$, satisfying the following properties:

(1) For any $z \in \Lambda_{\eta}$, the matrix $B_{\eta}(z)=C_{\eta}(F(z)) B(z) C_{\eta}^{-1}(z)$ has the Lyapunov block form

$$
\operatorname{diag}\left(B_{\eta, 1}(z), \cdots, B_{\eta, k}(z)\right),
$$

where each $B_{\eta, j}(z)$ is an $n_{j} \times n_{j}$ square matrix, $n_{j}$ denotes the multiplicity of the corresponding exponent $\lambda_{j}^{\prime}$ and the norm $\left\|B_{\eta, j}(z)\right\|$ and co-norm $\left\|B_{\eta, j}(z)^{-1}\right\|^{-1}$ lie in the interval $\left[e^{\lambda_{j}^{\prime}-\eta}, e^{\lambda_{j}^{\prime}+\eta}\right]$.

(2) There exists a measurable function $r_{1}: \Lambda_{\eta} \rightarrow\left(0, r^{\prime}\right]$, with

$$
e^{-\eta / \beta^{2}}<\frac{r_{1}(z)}{r_{1}(F(z))}<e^{\eta / \beta^{2}}, \quad \forall z \in \Lambda_{\eta}
$$

such that, for any point $z=(x, y) \in \Lambda_{\eta}$ and any $x^{\prime} \in B_{M}\left(x, r_{1}(z)\right)$, the map $h_{z^{\prime}, z}$ given by (24) where $z^{\prime}=\left(x^{\prime}, y\right)$, satisfies

$$
d_{C^{1}}\left(h_{z, z^{\prime}}, h_{z, z}\right) \leqslant \eta \text {. }
$$

(3) There exist measurable functions $K: \Lambda_{\eta} \rightarrow(0, \infty)$ and $r_{2}: \Lambda_{\eta} \rightarrow\left(0, r^{\prime}\right]$ with

$$
\begin{gathered}
e^{-\eta}<\frac{K(z)}{K(F(z))}<e^{\eta}, \\
e^{-\eta / \beta}<\frac{r_{2}(z)}{r_{2}(F(z))}<e^{\eta / \beta},
\end{gathered}
$$

such that, for every $z \in \Lambda_{\eta}$, and for any $y^{\prime}, y^{\prime \prime} \in \Phi_{z}^{-1}\left(B\left(0, r_{2}(z)\right)\right)$,

$$
\frac{1}{2} d_{N}\left(y^{\prime}, y^{\prime \prime}\right) \leqslant\left\|\Phi_{z}\left(y^{\prime}\right)-\Phi_{z}\left(y^{\prime \prime}\right)\right\| \leqslant K(z) d_{N}\left(y^{\prime}, y^{\prime \prime}\right)
$$

Proof. This result can be easily proved following mutatis mutandis the proof of [KH96, Theorem S.3.1]. The only significant difference is that to control our regular neighborhood we need two different radii, namely $r_{1}$ and $r_{2}$, for the size of the balls on the base space and on the fibers.

Then we recall some classical definitions of Pesin theory. Continuing with the notations of Theorem 4.1, every point $z=(x, y)$ of $\Lambda_{\eta}$ is called a regular point, and each set $B_{M}\left(x, r_{1}(z)\right) \times \Phi_{z}^{-1}\left(B\left(0, r_{2}(z)\right)\right)$ is called a regular neighborhood of $z$.

On the other hand, for each $\eta>0$ sufficiently small and any $\varepsilon>0$, by Luzin's theorem, there exist $\ell>0$ and a compact subset $\Lambda_{\eta, \ell} \subset \Lambda$ such that $\mu\left(\Lambda_{\eta, \ell}\right)>1-\varepsilon$, the functions $\left.r_{1}\right|_{\Lambda_{\eta, \ell}},\left.r_{2}\right|_{\Lambda_{\eta, \ell}},\left.K\right|_{\Lambda_{\eta, \ell}}$ and $\left.C_{\eta}\right|_{\Lambda_{\eta, \ell}}$ are continuous, and $\left|r_{1}(z)^{-1}\right| \leqslant \ell$ and $|K(z)| \leqslant \ell$, for any $z \in \Lambda_{\eta, \ell}$. The compact set $\Lambda_{\eta, \ell}$ is called the Pesin uniformity block of tolerance $\eta$ and bound $\ell$.

4.2. Fake Invariant Sets. For the sake of simplicity of the notation, given an arbitrary point $z_{0}=\left(x_{0}, y_{0}\right) \in M \times N$, we write $z_{n}=\left(x_{n}, y_{n}\right)=F^{n}\left(z_{0}\right)$, for every $n \in \mathbb{Z}$. Given any positive number $r$ we write

$$
U=U\left(z_{n}, r\right):=B_{M}\left(x_{n}, r\right) \times B_{N}\left(y_{n}, r\right),
$$

Continuing with the notation we introduced in $\$ 4$.1, let $\mu$ denote an ergodic $F$ invariant measure, $\eta$ be a sufficiently small positive number and $\ell$ a sufficiently large 
positive number, such that the Pesin uniformity block $\Lambda_{\eta, \ell} \subset \Lambda_{\eta}$ has positive $\mu$ measure. Hence by Poincaré recurrence theorem, for $\mu$-almost every point $z_{0} \in \Lambda_{\eta, \ell}$, there exists a positive integer number $N_{0}$ such that $z_{N_{0}} \in \Lambda_{\eta, \ell}$.

Now we can state the main result of this section:

Proposition 4.2 (Existence of fake invariant sets). There exist positive real constants $r^{(0)}, \kappa, C, \widetilde{C}$ such that, given any pair of points $z_{0}, z_{N_{0}} \in \Lambda_{\eta, \ell}$ (for some $\left.N_{0}>1\right)$, there exists a partition $\widehat{\mathcal{U}}^{n, s}$ of $U\left(z_{n}, r^{(n)}\right)$, for each $n \in\left\{0,1, \ldots, N_{0}\right\}$, where

$$
r^{(n)}:=r^{(0)} e^{-\frac{\eta}{\beta^{2}} \min \left\{n, N_{0}-n\right\}},
$$

satisfying the following properties:

(1) (Covering $f$-local stable sets) for any $z=(x, y) \in U\left(z_{n}, r^{(n)}\right)$, if $\widehat{\mathcal{U}}^{n, s}(z)$ denotes the atom of $\widehat{\mathcal{U}}^{n, s}$ containing $z$, then

$$
\operatorname{pr}_{1}\left(\widehat{\mathcal{U}}^{n, s}(z)\right)=W_{2 r^{(n)}}^{s}(x) \cap B_{M}\left(x_{n}, r^{(n)}\right) .
$$

(2) (Local invariance) for every $n \in\left\{0, \ldots, N_{0}-1\right\}$ and any $z \in U\left(z_{n}, \frac{r^{(n)}}{C}\right)$,

$$
F(z) \in U\left(z_{n+1}, r^{(n+1)}\right),
$$

and

$$
F\left(\widehat{\mathcal{U}}^{n, s}(z)\right) \cap U\left(z_{n+1}, r^{(n+1)}\right) \subset \widehat{\mathcal{U}}^{n+1, s}(F(z)) .
$$

(3) (Uniform contraction) for every $k \in\left\{1, \cdots, N_{0}\right\}$ and any pair of points $z^{\prime}, z^{\prime \prime} \in \widehat{\mathcal{U}}^{0, s}\left(z_{0}\right) \cap B_{M}\left(x_{0}, \frac{r^{(0)}}{C \widetilde{C}}\right) \times B_{N}\left(y_{0}, \frac{r^{(0)}}{C \widetilde{C}}\right)$,

$$
d\left(F^{k}\left(z^{\prime}\right), F^{k}\left(z^{\prime \prime}\right)\right) \leqslant \widetilde{C} e^{-k \kappa} d\left(z^{\prime}, z^{\prime \prime}\right) .
$$

The partitions $\widehat{\mathcal{U}}^{n, s}$ given by Proposition 4.2 will be called a fake locally stable foliations and their elements fake locally stable sets. Similarly, we can define the fake locally unstable foliations exhibiting analogous properties.

The rest of this section is devoted to the proof of Proposition 4.2. First we need some lemmas:

Lemma 4.3. Let $\mathbb{R}^{q}=\mathbb{R}^{u} \oplus \mathbb{R}^{c} \oplus \mathbb{R}^{s}$ be an orthogonal splitting and $L_{i}: \mathbb{R}^{q} \bigcirc$, with $i \in\{1, \ldots, n\}$, be linear maps that preserve the splitting. Suppose there exists $\lambda \in(0,1)$ such that it holds

$$
\begin{aligned}
& \max \left\{\left\|\left.L_{i}\right|_{\mathbb{R}^{s}}\right\|,\left\|\left(\left.L_{i}\right|_{\mathbb{R}^{u}}\right)^{-1}\right\|\right\}<\lambda ; \\
& \max \left\{\left\|\left.L_{i}\right|_{\mathbb{R}^{c}}\right\|,\left\|\left(\left.L_{i}\right|_{\mathbb{R}^{c}}\right)^{-1}\right\|\right\}<\lambda^{-\frac{1}{2}},
\end{aligned}
$$

for each $i \in\{1, \ldots, n\}$.

Then there exists a number $\alpha_{2}=\alpha_{2}(\lambda)>0$ such that if $f_{i}: \mathbb{R}^{q} \bigcirc$ are $C^{1}$ diffeomorphisms satisfying $d_{C^{1}}\left(f_{i}, L_{i}\right)<\alpha_{2}$ for every $i \in\{1, \ldots, n\}$, then there exists a family of foliations $\mathcal{W}_{i}^{s}$ of $\mathbb{R}^{q}$, called stable foliations, satisfying the following properties:

(1) $f_{i}\left(\mathcal{W}_{i}^{s}\right)=\mathcal{W}_{i+1}^{s}$, for each $i \in\{1, \ldots, n-1\}$;

(2) for any $x \in \mathbb{R}^{q}$, any $i \in\{1, \ldots, n-1\}$ and every $y \in \mathcal{W}_{i}^{s}(x)$,

$$
\left\|f_{i}^{k}(x)-f_{i}^{k}(y)\right\|<\lambda^{k}\|x-y\|, \quad \forall k \in\{1, \ldots, n-i\} .
$$

An analogous result holds for unstable foliations.

Proof. This is essentially Hadamard-Perron's theorem. See [KH96, Theorem 6.2.8] for details. 
Lemma 4.4. Let $\delta, \eta$ and $\ell$ be positive numbers and suppose that $e^{\eta}<\frac{4}{3}$. Let $f, g: \mathbb{R}^{q} \bigcirc$ be $C^{1}$-diffeomorphisms such that $d_{C^{1}}(f, g)<\delta$. Let $A, B \in \mathrm{GL}(q, \mathbb{R})$ be two linear transformations such that either $\|A\|<\ell e^{\eta}$ and $\|B\|<\ell$, or $\|A\|<\ell$ and $\|B\|<\ell e^{\eta}$. Then,

$$
d_{C^{1}}(A f B, A g B)<4 \ell^{2} \delta .
$$

Proof. The estimate easily follows from the following computation:

$$
\begin{aligned}
& d_{C^{1}}(\operatorname{AfB}(\cdot), \operatorname{AgB}(\cdot)) \\
= & d_{C^{0}}(\operatorname{AfB}(\cdot), \operatorname{AgB}(\cdot))+d_{C^{0}}(A \circ D f(B(\cdot)) \circ B(\cdot), A \circ D g(B(\cdot)) \circ B(\cdot)) \\
\leqslant & \|A\| d_{C^{0}}(f, g)+\|A\| d_{C^{0}}(D f, D g)\|B\|+\|A\| d_{C^{0}}(f, g)\|B\| \\
\leqslant & 3 \ell^{2} e^{\eta} \delta<4 \ell^{2} \delta .
\end{aligned}
$$

For any small number $r>0$, we can choose a $C^{1}$ map $\rho_{r}: \mathbb{R}^{q} \rightarrow[0,1]$ such that $\rho_{r}=1$ on $B(0, r)$ and $\rho_{r}=0$ on $\mathbb{R}^{q} \backslash B(0,2 r)$, and $\left\|\rho_{r}\right\|_{C^{0}} \leqslant 1,\left\|D \rho_{r}\right\|=O\left(\frac{1}{r}\right)$. Then, consider any $C^{1+\beta}$ function $f: N Ð$, where $N$ is a closed smooth Riemannian manifold. Define a diffeomorphism $f^{r}: T N \rightarrow T N$ as follows,

$$
f^{r}(x, v)=\rho_{r}(v) \times \exp _{f(x)}^{-1} \circ f \circ \exp _{x}(v)+\left(1-\rho_{r}(v)\right) \times D f_{x}(v) .
$$

Note that

$$
f^{r}(x, v):= \begin{cases}\exp _{f(x)}^{-1} \circ f \circ \exp _{x}(v), & \text { if }\|v\| \leqslant r \\ D f_{x}(v), & \text { if }\|v\| \geqslant 2 r .\end{cases}
$$

Then we have the following $C^{1}$ estimate for this diffeomorphism.

\section{Lemma 4.5 .}

$$
d_{C^{1}}\left(f^{r}(x, \cdot), D f_{x}(\cdot)\right)=O\left(r^{\beta}\right)
$$

Proof. By definition,

$$
f^{r}(x, v)-D f_{x}(v)=\rho_{r}(v) \times\left(\exp _{f(x)}^{-1} \circ f \circ \exp _{x}(v)-D f_{x}(v)\right) .
$$

Then, for $r$ small,

$$
\begin{aligned}
& \left\|f^{r}(x, \cdot)-D f_{x}(\cdot)\right\|_{C^{1}} \\
\leqslant & \left\|\rho_{r}(\cdot) \times\left(\exp _{f(x)}^{-1} \circ f \circ \exp _{x}(\cdot)-D f_{x}(\cdot)\right)\right\|_{C^{0}} \\
+ & \left\|D \rho_{r}(\cdot) \times\left(\exp _{f(x)}^{-1} \circ f \circ \exp _{x}(\cdot)-D f_{x}(\cdot)\right)\right\|_{C^{0}} \\
+ & \left\|\rho_{r}(\cdot) \times D\left(\exp _{f(x)}^{-1} \circ f \circ \exp _{x}(\cdot)-D f_{x}(\cdot)\right)\right\|_{C^{0}} \\
\leqslant & (2 r) C(2 r)^{\beta}+\frac{C}{r}(2 r) C^{\prime}(2 r)^{\beta}+C^{\prime}(2 r)^{\beta} \\
\leqslant & C\left(r^{1+\beta}+r^{\beta}+r^{\beta}\right)<C r^{\beta} .
\end{aligned}
$$

Proof of Proposition 4.2. Let $\lambda_{1}^{\prime}>\cdots>\lambda_{k}^{\prime}$ be the distinct fibered Lyapunov exponents of the linear cocycle $B$ defined in (22), with multiplicities $n_{1}, \cdots, n_{k}$, respectively.

Fix a number

$$
\kappa<\frac{1}{2} \min _{i}\left\{\lambda_{i}^{\prime}-\lambda_{i+1}^{\prime}, \tau\right\},
$$


where $\tau$ is the hyperbolicity rate of $f: M \rightarrow M$. With respect to the number $e^{-\kappa} \in(0,1)$, Lemma 4.3 gives the constant $\alpha_{2}$. Then we choose the number $\eta$ such that

$$
\eta<\min \left\{\beta^{2} \kappa, \frac{1}{2} \min _{i}\left\{\lambda_{i}^{\prime}-\lambda_{i+1}^{\prime}\right\}-\kappa, \frac{\alpha_{2}}{2}\right\} .
$$

Invoking Theorem 4.1, we know there is a measurable map $C_{\eta}: \Lambda_{\eta} \rightarrow \operatorname{GL}(q, \mathbb{R})$, a function $K: \Lambda_{\eta} \rightarrow(0,+\infty)$, with $K(z)>\max \left\{\left\|C_{\eta}(z)\right\|,\left\|C_{\eta}^{-1}(z)\right\|\right\}$, such that

$$
e^{-\eta}<\frac{K(z)}{K(F(z))}<e^{\eta}
$$

For any small $r>0, z=(x, y) \in \Lambda_{\eta}$, we can define $F_{z}^{r}$ as follows

$$
F_{z}^{r}\left(x^{\prime}, v\right)= \begin{cases}\exp _{\mathrm{pr}_{2} \circ F(z)}^{-1} \circ F\left(x^{\prime}, \exp _{y}(v)\right), & \text { if }\|v\|<r, \\ D\left(A\left(x^{\prime}\right)\right)(y)(v), & \text { if }\|v\|>2 r .\end{cases}
$$

For sufficiently $r>0$ sufficiently small $B(x, r) \subset M, F_{z}^{r}$ is well-defined on $B(x, r) \times$ $\mathbb{R}^{q}$. Moreover, by Lemma 4.5 , for some constant $K$,

$$
\left\|F_{z}^{r}(x, \cdot)-B(z)(\cdot)\right\|_{C^{1}} \leqslant K r^{\beta} .
$$

Note that for some $\ell$ sufficiently large, the uniformity block $\Lambda_{\eta, \ell} \subset \Lambda_{\eta}$ has positive $\mu$-measure. For any orbit segment $\left\{z_{0}, \cdots, z_{N_{0}}\right\}$, with $z_{0}, z_{N_{0}} \in \Lambda_{\eta, \ell}$, Theorem 4.1 item (1) gives $N_{0}$ linear maps

$$
B_{\eta}\left(z_{0}\right), B_{\eta}\left(z_{1}\right), \cdots, B_{\eta}\left(z_{N_{0}-1}\right)
$$

all with Lyapunov block forms.

Now we take

$$
r^{(0)}<\min \left\{\frac{1}{\ell^{2}},\left(\frac{\alpha_{2}}{8 K \ell^{2}}\right)^{1 / \beta}\right\}
$$

Then by (40),

$$
\left\|F_{z_{0}}^{r^{(0)}}\left(x_{0}, \cdot\right)-B\left(z_{0}\right)(\cdot)\right\|_{C^{1}}<\alpha_{2} / 8 \ell^{2},
$$

and by our choice of the uniformity block,

$$
\begin{aligned}
& \max \left(\left\|C_{\eta}\left(z_{0}\right)\right\|,\left\|C_{\eta}\left(z_{0}\right)^{-1}\right\|\right) \leqslant K\left(z_{0}\right) \leqslant \ell, \\
& \max \left(\left\|C_{\eta}\left(z_{1}\right)\right\|,\left\|C_{\eta}\left(z_{1}\right)^{-1}\right\|\right) \leqslant K\left(z_{1}\right) \leqslant \ell e^{\eta} .
\end{aligned}
$$

By Lemma 4.4, we get

$$
\left\|C_{\eta}\left(z_{1}\right) \circ F_{z_{0}}^{r^{(0)}}\left(x_{0}, \cdot\right) \circ C_{\eta}^{-1}\left(z_{0}\right)-B_{\eta}\left(z_{0}\right)\right\|_{C^{1}}<\alpha_{2} / 2 .
$$

So, it follows from Theorem 4.1 item (2) that, for any $x^{\prime} \in B\left(x_{0}, r^{(0)}\right)$, it holds

$$
\left\|C_{\eta}\left(z_{1}\right) \circ F_{z_{0}}^{r^{(0)}}\left(x^{\prime}, \cdot\right) \circ C_{\eta}^{-1}\left(z_{0}\right)-B_{\eta}\left(z_{0}\right)\right\|_{C^{1}}<\alpha_{2} .
$$

Now, let us consider the map $F_{z_{n}}^{r^{(n)}}\left(x_{n}, \cdot\right)$, for $n \in\left\{1, \ldots, N_{0}-1\right\}$.

By definition of $r^{(n)}$ in (28), and noting (25), it follows that for any $n=$ $0, \cdots, N_{0}, U\left(z_{n}, r^{(n)}\right)$ is contained in the regular neighborhood $B_{M}\left(x_{n}, r_{1}(z)\right) \times$ $\Phi_{z_{n}}^{-1}\left(B\left(0, r_{2}(z)\right)\right)$. Moreover,

$$
\begin{aligned}
r^{(n)} & <r^{(0)} e^{-\frac{\eta}{\beta} \min \left\{n, N_{0}-n\right\}} \\
& <\left(\frac{\alpha_{2}}{8 K \ell^{2}}\right)^{\frac{1}{\beta}} e^{-\frac{\eta}{\beta} \min \left\{n, N_{0}-n\right\}}=\left(\frac{\alpha_{2}}{8 K \ell^{2} e^{\eta \min \left\{n, N_{0}-n\right\}}}\right)^{\frac{1}{\beta}} .
\end{aligned}
$$

By Lemma 4.5,

$$
\left\|F_{z_{n-1}}^{r^{(n-1)}}\left(x_{n-1}, \cdot\right)-B\left(z_{n-1}\right)(\cdot)\right\|_{C^{1}}<\frac{\alpha_{2}}{8 \ell^{2}} e^{-\eta \min \left\{n, N_{0}-n\right\}} .
$$


Noting that $K\left(z_{n-1}\right) \leqslant \ell e^{\eta \min \left\{n, N_{0}-n\right\}}$ and $K\left(z_{n}\right) \leqslant \ell e^{\eta \min \left\{n+1, N_{0}-n-1\right\}}$, and applying Lemma 4.4, we obtain

$$
\left\|C_{\eta}\left(z_{n}\right) \circ F_{z_{n-1}}^{r^{(n-1)}}\left(x_{n-1}, \cdot\right) \circ C_{\eta}^{-1}\left(z_{n-1}\right)-B_{\eta}\left(z_{n-1}\right)\right\|_{C^{1}}<\frac{\alpha_{2}}{2},
$$

Then it follows from Theorem 4.1 that for any $x^{\prime} \in B\left(x_{n-1}, r^{(n-1)}\right)$,

$$
\left\|C_{\eta}\left(z_{n}\right) \circ F_{z_{n-1}}^{r^{(n-1)}}\left(x^{\prime}, \cdot\right) \circ C_{\eta}^{-1}\left(z_{n-1}\right)-B_{\eta}\left(z_{n-1}\right)\right\|_{C^{1}}<\alpha_{2} .
$$

Now, by Lemma 4.3 and the above estimates, the mappings $\left\{C_{\eta}\left(z_{n}\right) \circ F_{z_{n-1}}^{r^{(n-1)}}\left(x^{\prime}, \cdot\right) \circ\right.$ $\left.C_{\eta}^{-1}\left(z_{n-1}\right)\right\}_{n=1}^{N_{0}}$ admit stable foliations of $\mathbb{R}^{d}$. We write these stable foliations as $\mathcal{W}^{n-1, s, x^{\prime}}$.

The choice of $\eta$ in (37) gives $2 \kappa+2 \eta<\min _{i}\left\{\lambda_{i}^{\prime}-\lambda_{i+1}^{\prime}\right\}$. For any $w \in \mathcal{W}^{n, s, x_{n}^{\prime}}(v)$, where $x_{n}^{\prime} \in B\left(x_{n}, r^{(n)}\right)$, the forward iterates of $x_{n}^{\prime}$ will be denoted by $x_{n+1}^{\prime}, \cdots, x_{j}^{\prime}$. For $N_{0} \geqslant j \geqslant n$,

$$
\begin{aligned}
& d_{E}\left(C_{\eta}\left(z_{j+1}\right) \circ F_{z_{j}}^{r^{(j)}}\left(x_{j}^{\prime}, \cdot\right) \circ \cdots \circ F_{z_{n}}^{r^{(n)}}\left(x_{n}^{\prime}, \cdot\right) \circ C_{\eta}\left(z_{n}\right)^{-1}(w),\right. \\
& \left.C_{\eta}\left(z_{j+1}\right) \circ F_{z_{j}}^{r^{(j)}}\left(x_{j}^{\prime}, \cdot\right) \circ \cdots F_{z_{n}}^{r^{(n)}}\left(x_{n}^{\prime}, \cdot\right) \circ C_{\eta}\left(z_{n}\right)^{-1}(v)\right) \\
& \quad<e^{-2(j-n) \kappa} d_{E}(w, v),
\end{aligned}
$$

where we use $d_{E}$ to denote the standard Euclidean distance.

We can now define the stable partition $\widehat{\mathcal{U}}^{n, s}$ of $U\left(z_{n}, r^{(n)}\right)$. It is done in two steps. Firstly, we consider the stable foliation $W^{s}$ of the ball $B\left(x_{n}, r^{(n)}\right) \subset M$. Then, on each fiber over $x \in B\left(x_{n}, r^{(n)}\right)$, the set $B\left(y_{n}, r^{(n)}\right)$ is foliated by the the projection of the foliation $\mathcal{W}^{n, s, x}$ constructed above, i.e., the foliation $\exp _{y_{n}} \circ C_{\eta}\left(z_{n}\right)^{-1}\left(\mathcal{W}^{n, s, x}\right)$ restricted to $B\left(y_{n}, r^{(n)}\right)$. Note item (1) of the proposition follows directly from this definition.

For all $n=0, \cdots, N_{0}, r^{(n+1)} \geqslant r^{(n)} e^{-\frac{\eta}{\beta^{2}}}$, it is possible to choose a large constant $C$, such that

$$
F\left(U\left(z_{n}, \frac{r^{(n)}}{C}\right)\right) \subset U\left(z_{n+1}, r^{(n+1)}\right)
$$

This shows Item (2).

Now we prove item (3). Define $\widetilde{C}=\max \left\{\frac{\ell^{2}}{2}, K_{0}\right\}$, where $K_{0}$ is the constant in Definition 2.1. Now we take points $z^{\prime}, z^{\prime \prime} \in \widehat{\mathcal{U}}^{0, s}\left(z_{0}\right) \cap B_{M}\left(x_{0}, \frac{r^{(0)}}{C \widetilde{C}}\right) \times B_{N}\left(y_{0}, \frac{r^{(0)}}{C \widetilde{C}}\right)$, and we will write $z_{k}^{\prime}=\left(x_{k}^{\prime}, y_{k}^{\prime}\right)=F^{k}\left(z^{\prime}\right), z_{k}^{\prime \prime}=\left(x_{k}^{\prime \prime}, y_{k}^{\prime \prime}\right)=F^{k}\left(z^{\prime \prime}\right)$ for all $k$. Inductively, both points $z_{k}^{\prime}$ and $z_{k}^{\prime \prime}$ are contained in $\widehat{\mathcal{U}}^{k, s}\left(z_{k}\right) \cap B_{M}\left(x_{k}, \frac{r^{(k)}}{C}\right) \times$ $B_{N}\left(y_{k}, \frac{r^{(k)}}{C}\right)$. Then item (2) ensures the estimate (52) can be applied at each step.

Thus, by hyperbolicity of the base dynamics,

$$
d_{M}\left(x_{k}^{\prime}, x_{k}^{\prime \prime}\right) \leqslant K_{0} e^{-\tau k} d_{M}\left(x_{0}^{\prime}, x_{0}^{\prime \prime}\right) \leqslant \frac{r^{(k)}}{C}
$$

The second coordinate has a similar estimate. Indeed, it follows from (52) that, for any $k=1, \cdots, N_{0}-1$,

$$
d\left(y_{k}^{\prime}, y_{k}^{\prime \prime}\right) \leqslant d\left(y_{k}^{\prime}, y_{k}\right)+d\left(y_{k}^{\prime \prime}, y_{k}\right)
$$


We estimate

$$
\begin{aligned}
d\left(y_{k}^{\prime}, y_{k}\right) \leqslant & \ell e^{k \eta} d_{E}\left\{C_{\eta}\left(z_{k}\right) \circ F_{z_{k-1}}^{r^{(k-1)}}\left(x_{k-1}^{\prime}, \cdot\right) \circ \cdots \circ F_{z_{0}}^{r^{(0)}}\left(x_{0}^{\prime}, \exp _{y_{0}}^{-1}\left(y_{0}^{\prime}\right)\right)\right. \\
& \left.C_{\eta}\left(z_{k}\right) \circ F_{z_{k-1}}^{r^{(k-1)}}\left(x_{k-1}, \cdot\right) \circ \cdots \circ F_{z_{0}}^{r^{(0)}}\left(x_{0}, 0\right)\right\} \\
\leqslant & \ell e^{-k \kappa} d\left(C_{\eta}\left(z_{0}\right) \circ \exp _{y_{0}}^{-1}\left(y_{0}^{\prime}\right), 0\right) \\
\leqslant & \ell^{2} e^{-k \kappa} d\left(y_{0}^{\prime}, y_{0}\right) \leqslant \frac{r^{(k)}}{2 C}
\end{aligned}
$$

The term $d\left(y_{k}^{\prime \prime}, y_{k}\right)$ satisfies a similar estimate. So we have $d\left(y_{k}^{\prime}, y_{k}^{\prime \prime}\right) \leqslant \frac{r^{(k)}}{C}$. Combining the first and the second coordinate estimates, we have finished the proof of item (3) of the proposition.

\section{Fiber Closing LEMmA AND PROOF OF MAIN RESUltS}

In this section we prove Theorem 1.1. We start with a technical result which is some kind of "Closing Lemma along the fibers".

Let $F: M \times N \bigcirc$ be the skew-product induced by the hyperbolic homeomorphism $f: M ๖$ and the $C^{\beta}$-cocycle $A: M \rightarrow \operatorname{Diff}^{1+\beta}(N)$. Let $\mu$ be an ergodic $F$ invariant probability measure. Let $\eta, \ell$ be positive numbers with $\ell$ large enough in order to guarantee that the Pesin uniformity block $\Lambda_{\eta, \ell}$ of $F$ has positive $\mu$-measure. Let $r^{(0)}, C, \widetilde{C}, \kappa$ be the constants given by Proposition 4.2. In what follows, when there is no confusion, we denote the iterates of a point $z_{0}=\left(x_{0}, y_{0}\right) \in M \times N$ by $z_{i}=\left(x_{i}, y_{i}\right)=F^{i}\left(z_{0}\right)$, for every $i \in \mathbb{Z}$. Since $\mu\left(\Lambda_{\eta, \ell}\right)>0$, notice that by Poincaré recurrence theorem, there exists $z_{0} \in \Lambda_{\eta, \ell}$ such that $z_{n}$ belongs to $\Lambda_{\eta, \ell}$ too, for some $n \geqslant 1$.

Lemma 5.1. There exist constants $\varepsilon_{0}, K>0$ such that for any point $z_{0} \in \Lambda_{\eta, \ell}$ such that there is $n \geqslant 1$ with $z_{n} \in \Lambda_{\eta, \ell}$ and $d\left(z_{0}, z_{n}\right)<\varepsilon_{0}$, there is some F-orbit segment $\left\{z_{0}^{\prime \prime}, z_{1}^{\prime \prime}, \cdots, z_{n}^{\prime \prime}\right\}$, with $p r_{1}\left(z_{0}^{\prime \prime}\right)=p r_{1}\left(z_{n}^{\prime \prime}\right)$ and satisfying

$$
d\left(z_{i}^{\prime \prime}, z_{i}\right) \leqslant K d\left(z_{0}, z_{n}\right) e^{-\kappa \min \{i, n-i\}} .
$$

Proof. Let us define $K=\max \{\ell, 2 C \widetilde{C}\}$ and $\varepsilon_{0}=r^{(0)} / K$.

First note that for any orbit segment satisfying $d\left(z_{0}, z_{n}\right)<\varepsilon_{0}$ and $z_{0}, z_{n} \in \Lambda_{\eta, \ell}$, the point $z_{n}$ lies in the regular neighborhood of $z_{0}$.

In particular, $d\left(x_{0}, x_{n}\right)<\varepsilon_{0}$ and hence we can apply Anosov Closing Lemma to conclude there is a unique periodic point $p$ with period $n$ which exponentially shadows the orbit segment $\left\{x_{0}, \cdots, x_{n}\right\}$. Consider the point $x_{0}^{\prime}=W^{s}\left(x_{0}\right) \cap W^{u}(p)$. Then, for each $i=0, \cdots, n$ it holds

$$
d\left(x_{i}, x_{i}^{\prime}\right)<\frac{K}{2} e^{-\tau \min \{i, n-i\}} d\left(x_{0}, x_{n}\right) .
$$

Since $x_{0}^{\prime} \in W_{\frac{r^{(0)}}{K}}^{s}\left(x_{0}\right)$, by Proposition 4.2, $x_{0}^{\prime}$ has a pre-image by $\mathrm{pr}_{1}$ which is contained in the local fake stable set of $z_{0}$. Denote this point by $z_{0}^{\prime} \in \widehat{\mathcal{U}}^{0, s}\left(z_{0}\right)$. Moreover, by our choice of $K$ it holds

$$
d\left(z_{i}, z_{i}^{\prime}\right)<\frac{K}{2} d\left(z_{0}, z_{0}^{\prime}\right) e^{-\kappa \min \{i, n-i\}}, \quad \forall i \in\{1, \cdots, n\}
$$

In particular, $d\left(z_{n}^{\prime}, z_{n}\right)<r^{(n)}$. Notice that $r^{(n)}=r^{(0)}$, too.

Now let us consider the point $x_{n}^{\prime}=\operatorname{pr}_{1}\left(z_{n}^{\prime}\right) \in M$. Since $d\left(p, x_{n}^{\prime}\right) \leqslant d\left(p, x_{n}\right)+$ $d\left(x_{n}, x_{n}^{\prime}\right)$, the periodic point $p \in W_{2 \varepsilon_{0}}^{u}\left(x_{n}^{\prime}\right)$. Applying Proposition 4.2 in the backward direction, we conclude the point $p$ has a pre-image under $\mathrm{pr}_{1}$, denoted as 
$z_{n}^{\prime \prime} \in \widehat{\mathcal{U}}^{n, u}\left(z_{n}^{\prime}\right)$, such that if we write $z_{i}^{\prime \prime}:=F^{-(n-i)}\left(z_{n}^{\prime \prime}\right)$ for all $i=0, \cdots, n-1$, then it holds

$$
d\left(z_{i}^{\prime \prime}, z_{i}^{\prime}\right)<\frac{K}{2} d\left(z_{n}^{\prime \prime}, z_{n}^{\prime}\right) e^{-\kappa \min \{i, n-i\}} .
$$

Combing both (58) and (59), for all $i=0, \cdots, n$,

$$
d\left(z_{i}^{\prime \prime}, z_{i}\right) \leqslant d\left(z_{i}^{\prime \prime}, z_{i}^{\prime}\right)+d\left(z_{i}^{\prime}, z_{i}\right) \leqslant K d\left(z_{n}, z_{0}\right) e^{-\kappa \min \{i, n-i\}},
$$

where the last inequality is consequence of the fact that $d\left(z_{0}, z_{n}\right) \sim d\left(z_{0}, z_{0}^{\prime}\right)$ and $d\left(z_{0}, z_{n}\right) \sim d\left(z_{n}^{\prime \prime}, z_{n}^{\prime}\right)$, maybe increasing $K$ if it is necessary. Finally it clearly holds $\operatorname{pr}_{1}\left(z_{n}^{\prime \prime}\right)=\operatorname{pr}_{1}\left(z_{0}^{\prime \prime}\right)=p$.

Proof of Theorem 1.1. According to Theorem 3.1 of [KP16], under the periodic orbit condition (3), it suffices to show that the fiber-wise Lyapunov exponents all vanish, with respect to any ergodic $F$-invariant probability measure $\mu$.

Reasoning by contradiction, suppose that there exists an ergodic $F$-invariant measure $\mu$ such that their fibered Lyapunov exponents, listed by $\lambda_{1} \geqslant \cdots \geqslant \lambda_{q}$, are not all equal to zero. If the measure is supported on finitely many fibers, then we immediately get a contradiction, because the periodic orbit condition clearly implies all Lyapunov exponents vanish. If this is not the case, we consider a positive number $\delta$ which is smaller than the absolute value of one non-vanishing Lyapunov exponent.

We claim that there exists an orbit segment $z_{0}^{\prime \prime}, \cdots, z_{n}^{\prime \prime}$ with $\operatorname{pr}_{1}\left(z_{0}^{\prime \prime}\right)=\operatorname{pr}_{1}\left(z_{n}^{\prime \prime}\right)$, such that, if we denote the singular values of $D A^{(n)}\left(x_{0}^{\prime \prime}\right)\left(y_{0}^{\prime \prime}\right)$ by $e^{n \sigma_{1}} \geqslant \cdots \geqslant e^{n \sigma_{d}}$, then they satisfy $\left|\sigma_{j}-\lambda_{j}\right| \leqslant \delta$ for all $1 \leqslant j \leqslant d$. In such a case, this linear map has at least one non-vanishing singular value, contradicting the periodic orbit condition (3).

So, it just remains to prove our claim. Let $\kappa$ be a positive real number satisfying estimate (36), and let $\alpha_{1}=\alpha_{1}(\delta / 2)$ be the positive constant given by Lemma 3.3. Then, we apply Lemma 4.3 getting a new constant $\alpha_{2}:=\alpha\left(e^{-\kappa}\right)$; and we take $\alpha:=\min \left\{\alpha_{1}, \alpha_{2}\right\}$. Thus, let $\eta$ be a positive number satisfying (37). By Theorem 4.1, we can choose a uniformity block $\Lambda_{\eta, \ell}$ with positive $\mu$-measure. Finally, let $\varepsilon_{0}$ and $K$ be the positive constants given by Lemma 5.1 .

Then, we choose a point $z$ in $\Lambda_{\eta, \ell}$ which is a $\mu$-density point of $\Lambda_{\eta, \ell}$ and consequently, the ball $B\left(z, \varepsilon_{0} / 2\right)$ intersects $\Lambda_{\eta, \ell}$ with positive $\mu$-measure. By Poincaré's recurrence theorem, we can assume there are infinitely many natural numbers $n$ such that the $F$-orbit of $z$ satisfies $z_{0}, z_{n} \in B\left(z, \varepsilon_{0} / 2\right) \cap \Lambda_{\eta, \ell}$.

Applying Lemma 5.1, there is an $F$-orbit segment $z_{0}^{\prime \prime}, z_{1}^{\prime \prime}, \cdots, z_{n}^{\prime \prime}$, with $\operatorname{pr}_{1}\left(z_{0}^{\prime \prime}\right)=$ $\operatorname{pr}_{1}\left(z_{n}^{\prime \prime}\right)$, such that,

$$
d\left(z_{i}, z_{i}^{\prime \prime}\right)<K d\left(z_{0}, z_{n}\right) e^{-\kappa \min \{i, n-i\}} \text { for all } i=0, \cdots, n .
$$

Thus, by formula (50) we know that

$$
\left\|C_{\eta}\left(z_{i}\right) \circ F_{z_{i-1}}^{r^{(i-1)}}\left(z_{i-1}, \cdot\right) \circ C_{\eta}^{-1}\left(z_{i-1}\right)^{-1}-B_{\eta}\left(z_{i-1}\right)\right\|_{C^{1}}<\alpha .
$$

So, if $\tilde{\sigma}_{1}^{(n)} \geqslant \tilde{\sigma}_{2}^{(n)} \geqslant \ldots \geqslant \tilde{\sigma}_{q}^{(n)}$ denotes the singular values of the linear map $C_{\eta}\left(z_{n}\right) \circ D A^{n}\left(x_{0}^{\prime \prime}\right)\left(y_{0}^{\prime \prime}\right) \circ C_{\eta}\left(z_{0}\right)^{-1}$, then invoking Lemma 3.3 for the constant $\delta / 2$, we can guarantee that

$$
\left|\frac{1}{n} \log \tilde{\sigma}_{j}^{(n)}-\lambda_{j}\right|<\frac{\delta}{2}
$$

for each $i \in\{1, \ldots, q\}$.

Finally, note that the changes of Lyapunov coordinates at $z_{0}$ and $z_{n}$, namely $C_{\eta}\left(z_{0}\right)$ and $C_{\eta}\left(z_{n}\right)$ and their inverses, are bounded by $\ell$. So we can apply Lemma 3.2 
to conclude that, if $\sigma_{1}^{(n)} \geqslant \sigma_{2}^{(n)} \geqslant \ldots \geqslant \sigma_{q}^{(n)}$ are the singular values of the linear map $D A^{n}\left(x_{0}^{\prime \prime}\right)\left(y_{0}^{\prime \prime}\right)$, then it holds

$$
\left|\frac{1}{n} \log \tilde{\sigma}_{j}^{(n)}-\lambda_{j}\right|<\delta
$$

for every $i$, completing the proof of the theorem.

\section{REFERENCES}

[BW10] K. Burns and A. Wilkinson, On the ergodicity of partially hyperbolic systems, Annals of Mathematics (2010), 451-489.

[dlLW10] R. de la Llave and A. Windsor, Livšic theorems for non-commutative groups including diffeomorphism groups and results on the existence of conformal structures for anosov systems, Ergodic Theory Dynam. Systems 30 (2010), no. 4, 1055-1100. MR 2669410

[dlLW11] _ Smooth dependence on parameters of solutions to cohomology equations over anosov systems with applications to cohomology equations on diffeomorphism groups, Discrete Contin. Dyn. Syst. 29 (2011), no. 3, 1141-1154. MR 2773168

[Guy] M. Guysinsky, Livšic theorem for cocycles with values in the group of diffeomorphisms, in preparation.

[HPS77] M. W. Hirsch, C. C. Pugh, and M. Shub, Invariant manifolds, Lecture Notes in Mathematics, Vol. 583, Springer-Verlag, Berlin, 1977. MR 0501173

[Kal11] B. Kalinin, Livšic theorem for matrix cocycles, Ann. of Math. 173 (2011), no. 2, 10251042. MR 2776369

[KH96] A. Katok and B. Hasselblatt, Introduction to the modern theory of dynamical systems, vol. 54, Cambridge Univ. Pr., 1996.

[KK96] A. Katok and A. Kononenko, Cocycle stability for partially hyperbolic systems, Math. Res. Letters 3 (1996), 191-210.

[KP16] A. Kocsard and R. Potrie, Livšic theorem for low-dimensional diffeomorphism cocycles, Comment. Math. Helv. 91 (2016), 39-64.

[Liv71] A. N. Livšic, Certain properties of the homology of Y-systems, Mat. Zametki 10 (1971), $555-564$.

[Liv72a] Cohomology of dynamical systems, Izv. Akad. Nauk SSSR Ser. Mat. 36 (1972), $1296-1320$

[Liv72b] The homology of dynamical systems, Uspehi Mat. Nauk 27 (1972), no. 3(165), 203-204. MR 0394768

[NP13] A. Navas and M. Ponce, A Livšic type theorem for germs of analytic diffeomorphisms, Nonlinearity 26 (2013), no. 1, 297-305.

[NT95] V. Niţică and A. Török, Cohomology of dynamical systems and rigidity of partially hyperbolic actions of higher-rank lattices, Duke Math. J. 79 (1995), no. 3, 751-810.

[NT96] V. Niţică and A. Török, Regularity results for the solutions of the Livsic cohomology equation with values in diffeomorphism groups, Ergodic Theory Dynam. Systems 16 (1996), no. 2, 325-333.

[Wil13] A. Wilkinson, The cohomological equation for partially hyperbolic diffeomorphisms, Astérisque 358 (2013), 75-165. MR 3203217

(Avila) CNRS, IMJ-PRG, UMR 7586, Univ Paris Diderot, Sorbonne Paris Cite, SorBonnes Universités, UPMC Univ Paris 06, F-75013, Paris, France \& IMPA, Estrada Dona Castorina 110, Rio de Janeiro, Brasil

E-mail address: artur@math.univ-paris-diderot.fr

(Kocsard) Instituto de Matemática e Estatística - Universidade Federal Fluminense. Rua Professor Marcos Waldemar de Freitas Reis, s/n. Bloco H - Campus de Gragoatá. 24.210-201, NiteróI, RJ - BRASIL

E-mail address: akocsard@id.uff.br

(Liu) IMPA, Estrada Dona Castorina 110, Rio de Janeiro, Brasil

E-mail address: 1xc1984@impa.br 